\title{
Red Blood Cells for Glucose-Responsive Insulin Delivery
}

\author{
Chao Wang, Yanqi Ye, Wujin Sun, Jicheng Yu, Jingqiang Wang, David S. Lawrence, \\ John B. Buse, and Zhen Gu*
}

\begin{abstract}
Glucose-responsive delivery of insulin mimicking the function of pancreatic $\beta$-cells to achieve meticulous control of blood glucose (BG) would revolutionize diabetes care. Here the authors report the development of a new glucose-responsive insulin delivery system based on the potential interaction between the glucose derivative-modified insulin (Glc-Insulin) and glucose transporters on erythrocytes (or red blood cells, RBCs) membrane. After being conjugated with the glucosamine, insulin can efficiently bind to RBC membranes. The binding is reversible in the setting of hyperglycemia, resulting in fast release of insulin and subsequent drop of BG level in vivo. The delivery vehicle can be further simplified utilizing injectable polymeric nanocarriers coated with RBC membrane and loaded with Glc-Insulin. The described work is the first demonstration of utilizing RBC membrane to achieve smart insulin delivery with fast responsiveness.
\end{abstract}

Diabetes currently affects 415 million people worldwide and this number is expected to increase to 642 million by 2040..$^{[1,2]}$ Insulin is essential for survival in type 1 diabetes and often required for treatment of type 2 diabetes in order to control glycemia and prevent complications. ${ }^{[3,4]}$ However, traditional

\author{
Dr. C. Wang, Y. Ye, W. Sun, J. Yu, Dr. J. Wang, Prof. Z. Gu \\ Joint Department of Biomedical Engineering \\ University of North Carolina at Chapel Hill \\ and North Carolina State University \\ Raleigh, NC 27695, USA \\ E-mail: zgu@email.unc.edu \\ Dr. C. Wang, Y. Ye, W. Sun, J. Yu, Dr. J. Wang, Prof. Z. Gu \\ Division of Molecular Pharmaceutics and \\ Center for Nanotechnology in Drug Delivery \\ Eshelman School of Pharmacy \\ University of North Carolina at Chapel Hill \\ Chapel Hill, NC 27599, USA \\ Dr. D. S. Lawrence \\ Department of Chemistry \\ University of North Carolina \\ Chapel Hill, NC 27599, USA \\ Dr. D. S. Lawrence \\ Division of Chemical Biology and Medicinal Chemistry \\ University of North Carolina \\ Chapel Hill, NC 27599, USA \\ Dr. D. S. Lawrence \\ Department of Pharmacology \\ University of North Carolina \\ Chapel Hill, NC 27599, USA \\ Dr. J. B. Buse, Prof. Z. Gu \\ Department of Medicine \\ University of North Carolina School of Medicine \\ Chapel Hill, NC 27599, USA
}

DOI: 10.1002/adma.201606617 exogenous insulin administration cannot match the exquisite regulation of blood glucose (BG) achieved by $\beta$-cells within the pancreatic islets of Langerhans, where endogenous insulin secretion is linked through metabolism to glucose transport. Poor glucose control results in a high risk for diabetes complications, such as limb amputation, blindness, and kidney failure. In addition, hypoglycemia can lead to behavioral and cognitive disturbance, seizure, coma, brain damage, or death. "Smart" glucose-responsive insulin delivery devices or formulations, which can mimic the function of $\beta$-cells to regulate insulin "secretion," are therefore highly desirable with an aim to improve blood glucose control and quality of life for people with diabetes. ${ }^{[5,6]}$

Such smart therapies generally integrate a glucose-sensing or conversion module and a sensing/conversion-activated insulin releasing module. For example, the wearable closedloop electronic/mechanical pumps combine a continuous glucose-monitoring electrochemical sensor and an external insulin infusion pump. ${ }^{[4]}$ These systems have historically been limited by lag in blood glucose equilibration with the interstitium, insulin absorption into the circulation and biofouling. ${ }^{[7]}$ Synthetic materials-based glucose-responsive formulations have also been widely explored since the 1970s. ${ }^{[5,8-10]}$ Three classical strategies are often utilized, ${ }^{[6]}$ typically including different glucose-sensing moieties: glucose oxidase, ${ }^{[11-15]}$ glucosebinding proteins (e.g., ConA), ${ }^{[16]}$ and phenylboronic acid ${ }^{[17]}$ for achieving glucose triggers. A variety of formulations, such as bulk hydrogels, microgels, emulsion-based nanoparticles, and self-assembled vesicles have been developed to respond toward a hyperglycemic state to swell, ${ }^{[18,19]}$ shrink, ${ }^{[20]}$ degrade, ${ }^{[11,14]}$ or dissociate ${ }^{[13,21,22]}$ in order to promote the release of insulin. In spite of these promising strategies, it remains challenging to demonstrate a system that responds rapidly to elevated BG levels, closely mirroring the kinetics of a healthy pancreas. In addition, immunological responses, stability in the physiological environment and long-term toxicity of those synthetic systems require further investigation.

Here we report a new strategy for glucose-responsive insulin delivery based on the red blood cells (RBCs) or erythrocytes system. As illustrated in Figure 1, we found that the glucose derivative-modified insulin (designated Glc-Insulin) can effectively bind to the membrane of mouse and human RBCs. We think this occurs maybe via the glucose transporter (GLUT) molecule, an abundant membrane protein on RBCs. Such binding is reversible and insulin can be rapidly released 


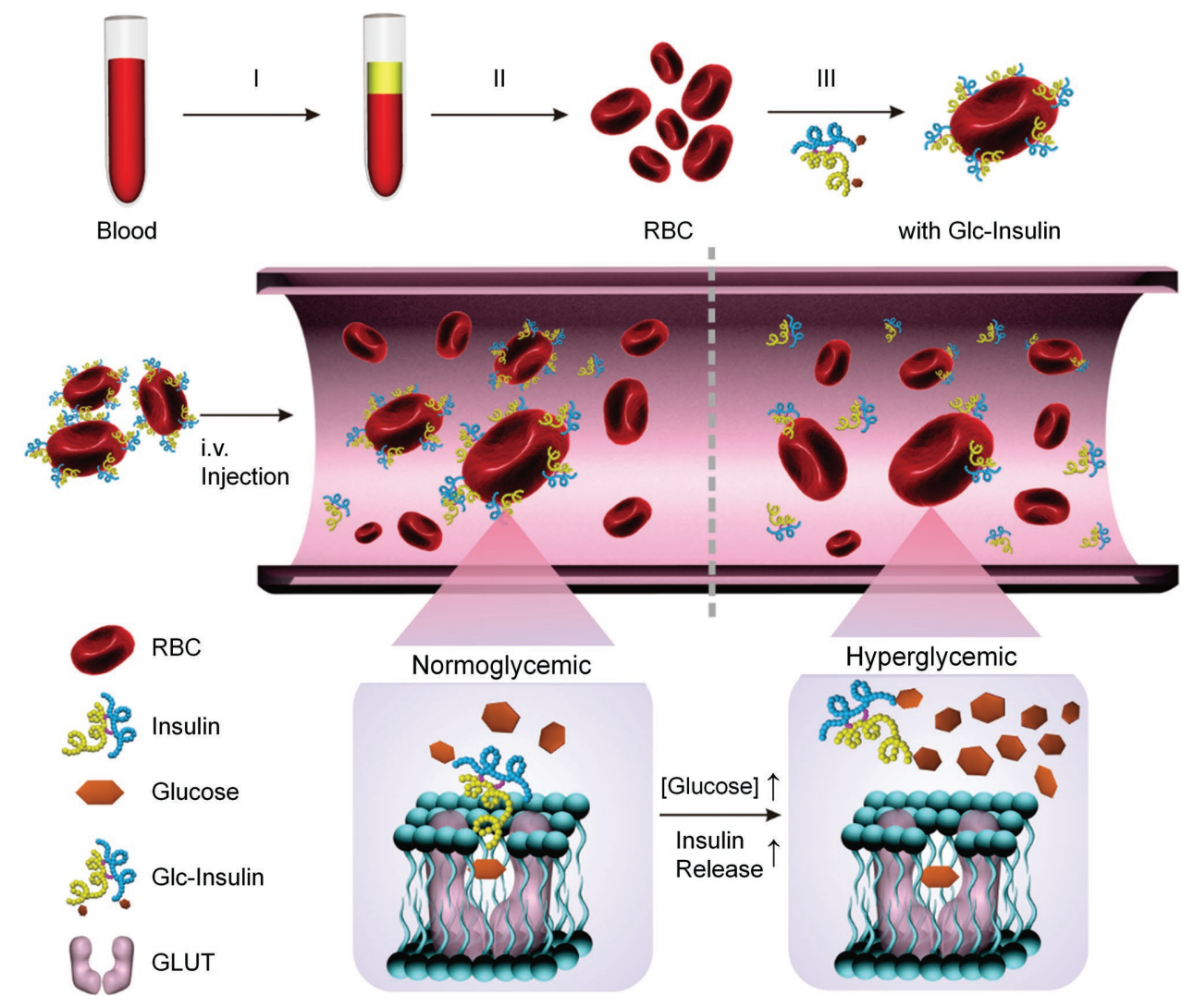

Figure 1. Schematic of the glucose-responsive insulin delivery system based on red blood cells. Synthesized Glc-Insulin was attached to erythrocytes by interacting with glucose receptor/transporter on plasma membranes. I,II) Collection and isolation of mRBCs. III) Binding of Glc-Insulin to mRBCs.

from RBCs under high glucose conditions, mediated by displacement due to competitive interaction of free glucose with GLUTs. As the most abundant cellular constituent in the blood, human RBCs have a life span of 100-120 days as natural carriers for oxygen in blood vessel. Importantly, inherent biocompatibility of RBCs makes them excellent candidates for robust glucose-response insulin delivery carriers. ${ }^{[23-29]}$ Using chemically induced type 1 mice as an animal model, we demonstrated that intravenous (i.v.) injection of mouse RBCs (mRBCs) coupled with Glc-Insulin greatly prolonged the therapeutic effect of insulin to maintain BG levels within the normal range.

Furthermore, this "glucose-responsive RBC" strategy can be extended by combining poly(lactic-co-glycolic acid) (PLGA) nanoparticles coated with RBC membranes (RM@PLGA). As with intact RBCs, Glc-Insulin can binds to RM@PLGA nanoparticles specifically. Insulin release from these nanoparticles is self-regulated in a BG-mediated manner. To our best knowledge, the described work is the first demonstration of utilizing RBCs membrane to realize glucose-responsive insulin delivery both in vitro and in vivo.

Glucosamine was conjugated to insulin to from Glc-Insulin via a bifunctional maleimide linker (Figure S1, Supporting Information). Many published studies have demonstrated that using glucose conjugates for targeting the GLUTs of cells. ${ }^{[30-36]}$ In our work, Glc-Insulin is clearly identified by the sodium dodecyl sulfate polyacrylamide gel electrophoresis and matrix-assisted laser desorption/ionization mass spectrometry
(MALDI-MS) (Figure S2, Supporting Information). As calculated by MALDI-MS, two glucose derivative molecules are conjugated to each insulin molecule. The circular dichroism spectra of native insulin and Glc-Insulin are virtually superimposable, indicating that the secondary structures of these species are similar if not identical (Figure S3A, Supporting Information). Most importantly, the Glc-Insulin conjugate and native insulin do not display any significant difference in their bioactivity profiles upon administration in streptozotocin (STZ)-induced type 1 diabetic mice (Figure S3B, Supporting Information). In short, the modification of insulin with glucose derivative has a statistically insignificant impact on bioactivity relative to unmodified insulin.

GLUTs are membrane proteins that facilitate the transport of glucose down a concentration gradient through the plasma membrane. ${ }^{[37]}$ Glucose transporter 1 (GLUT1) is the main glucose transporter on human RBCs; while glucose transporter 4 (GLUT4) is the dominant glucose transporter in RBCs of adult mice ${ }^{[38-40]}$ (Figure S4, Supporting Information). Meanwhile, glucosamine is structurally homologous to glucose and has also been shown to bind to GLUT1/GLUT4 transporters. ${ }^{[41,42]}$ The affinity for glucosamine and glucose of GLUT1 and GLUT4 are similar. ${ }^{[43,44]}$ In our study, we hypothesize that Glc-Insulin can bind to $\mathrm{mRBCs}$ via the specific glucosamineGLUT binding. The mRBCs were incubated with Glc-Insulin overnight and imaged using the confocal fluorescence microscope. Indeed, we observed effective binding of Glc-Insulin to 
A

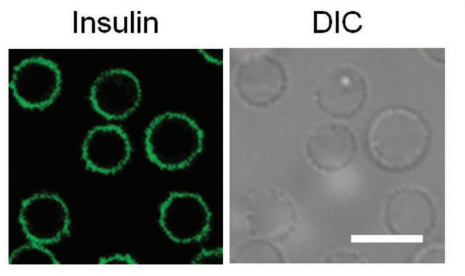

$\mathrm{D}$

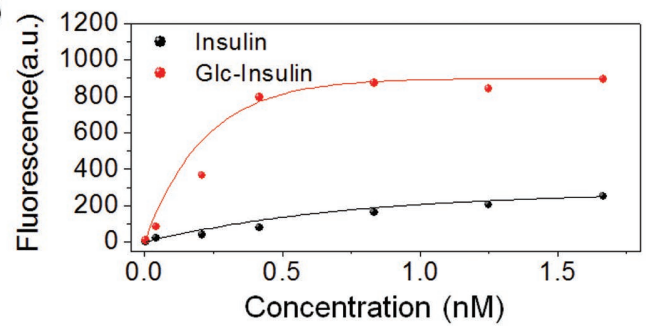

F

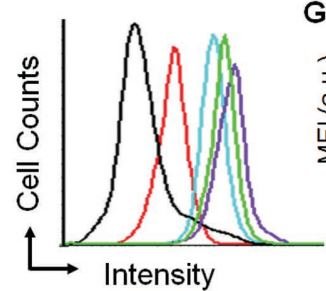

H

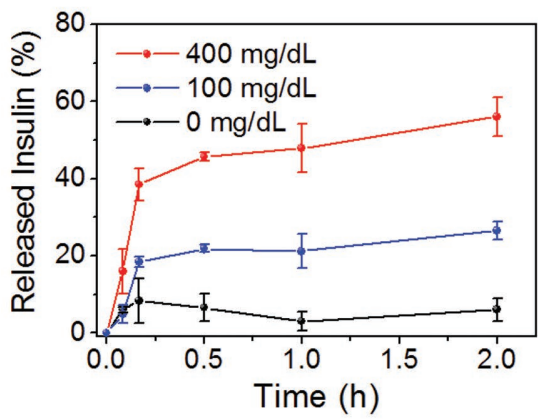

B

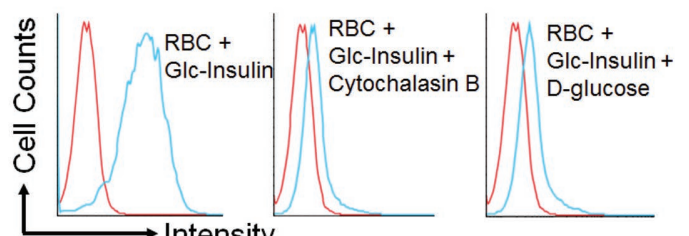

C
E
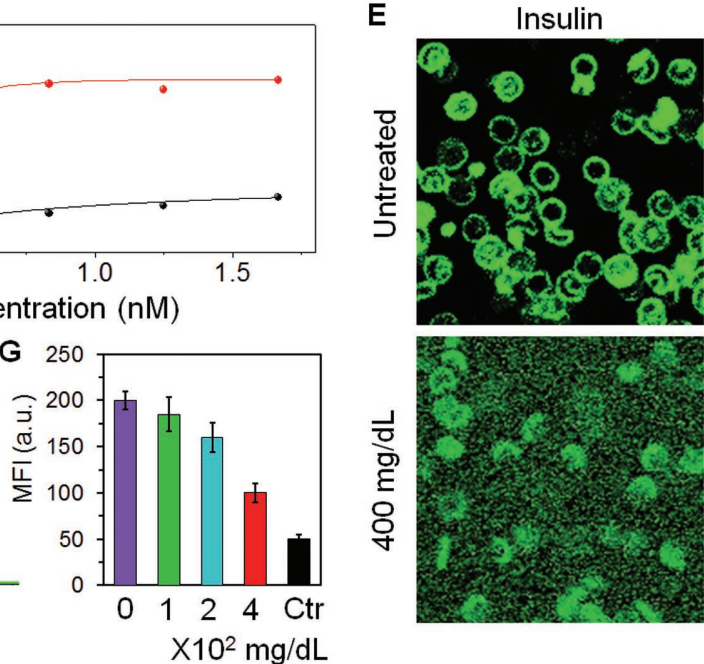

I

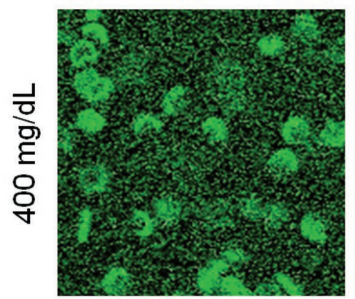

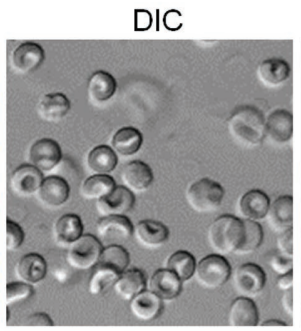
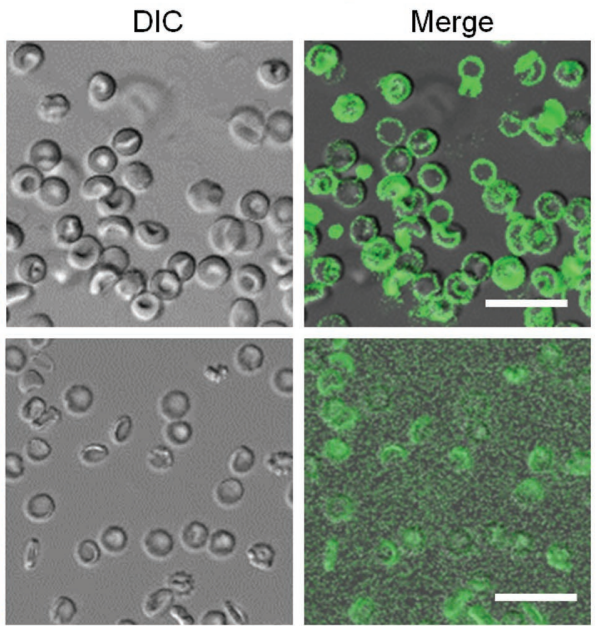

$\mathrm{J}$

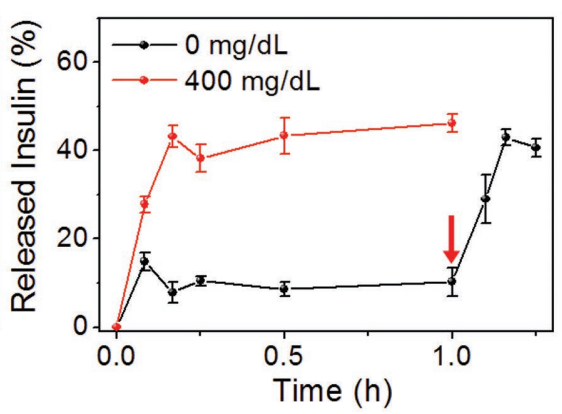

Figure 2. Characterization of the Glc-Insulin attached mRBCs. A) Confocal immunofluorescence imaging of the Glc-Insulin attached to mRBCs. Rat anti-human insulin FITC-conjugated antibody was used to detect insulin on mRBCs (scale bar $=10 \mu \mathrm{m}$ ). B) Flow cytometry analysis of mRBCs incubated with different experimental settings. The binding of glucose-insulin and RBCs was significantly inhibited by D-glucose and Cytochalasin B. C) Flow cytometry data of mRBCs incubated with insulin and Glc-insulin. D) Flow cytometric assay used to assess the specific binding of Glc-Insulin toward mRBCs. E) Confocal microscopy images of the Glc-Insulin attached mRBCs untreated and treated with $400 \mathrm{mg} \mathrm{dL}^{-1}$ glucose solution (scale bar $=50 \mu \mathrm{m}$ ). F,G) Flow cytometry data of insulin attached mRBCs treated with different concentration of glucose solutions and corresponding mean fluorescent intensity (MFI) quantification of insulin-mRBCs (Ctr means naïve mRBCs). $\mathrm{H}-\mathrm{J}$ ) In vitro glucose-responsive insulin release. H) In vitro accumulated Glc-Insulin release from the mRBCs in several glucose concentrations as indicate at $37^{\circ} \mathrm{C}$. I) In vitro accumulated Glc-Insulin release from the mRBCs. The arrow indicates the glucose ( $400 \mathrm{mg} \mathrm{mL}^{-1}$ ) administration points. J) Pulsatile release profile of mRBCs presents the rate of insulin release as a function of glucose concentration (blue: $100 \mathrm{mg} \mathrm{dL}-1$ and red: $400 \mathrm{mg} \mathrm{dL}^{-1}$ ). The error bars are based on the standard deviation (SD) of triplicated samples.

mRBCs (Figure 2A). Importantly, this binding of Glc-insulin toward RBCs can be significantly inhibited by D-glucose and Cytochalasin $\mathrm{B},{ }^{[35]}$ suggesting the GLUT-mediated interaction (Figure 2B). We further compared the Glc-Insulin with native insulin. The flow-cytometry results suggested that the attachment of Glc-Insulin toward mRBCs was via the specific interaction. With modification of the glucose derivative, insulin more easily attached to mRBCs than native insulin (Figure 2C). While insignificant binding of free insulin toward mRBCs was observed (Figure 2D). The loaded insulin to mRBCs was determined by the enzyme-linked immunosorbent assay (ELISA). mRBCs could be readily coupled with Glc-Insulin about $5 \times 10^{-16} \mathrm{~g}$ per cell. Furthermore, the annexin $\mathrm{V}$ binding assay confirmed that insulin binding did not cause significant damage to mRBCs (Figure S5, Supporting Information).

To examine the glucose-responsive insulin release performance, Glc-Insulin attached mRBCs were incubated in phosphate-buffered saline (PBS) containing different concentrations of glucose. Rat anti-human insulin fluorescein isothiocyanate (FITC)-conjugated antibody was used to label the bound 
insulin. As observed in confocal imaging (Figure 2E), compared to untreated insulin-mRBCs, the fluorescence signals of insulin on the mRBCs membrane were notably decreased after 30 min when treated with $400 \mathrm{mg} \mathrm{dL}^{-1}$ glucose in PBS, due to the detachment of the insulin from mRBCs (Figure 2E). The flow-cytometry results further substantiated that the signals of insulin on $\mathrm{mRBCs}$ decreased with increased glucose concentration. Only $\approx 50 \%$ less insulin signals was detected on $400 \mathrm{mg} \mathrm{dL}^{-1}$ glucose treated mRBCs (Figure 2F,G). We next assessed the insulin release profile in response to varying glucose levels, including a typical hyperglycemic level (400 $\mathrm{mg} \mathrm{dL}^{-1}$ ), normoglycemic levels (100 and $200 \mathrm{mg} \mathrm{dL}^{-1}$ ), and a control level $\left(0 \mathrm{mg} \mathrm{dL} \mathrm{L}^{-1}\right)$. The released amounts of insulin from mRBCs were determined by the ELISA assay. As shown in Figure $2 \mathrm{H}$, about $50 \%$ of insulin was released from $\mathrm{mRBCs}$ incubated with $400 \mathrm{mg} \mathrm{dL}^{-1}$ glucose within $2 \mathrm{~h}$, significantly higher than samples associated with normal glucose levels. These results were also consistent with the flow cytometry data mentioned above. Importantly, the quick release of insulin after exposure to high glucose was documented (Figure 2I), suggesting the potential for clinical effectiveness. Furthermore, pulsatile release kinetics of insulin was achieved when the glucose concentration was periodically varied between the normal and hyperglycemic levels for several cycles (Figure 2J). The release rates increased to a maximum within the first $10 \mathrm{~min}$ and then gradually decreased, which can be attributed to the gradual depletion of Glc-Insulin interacting with GLUTs.

Next, we examined the glucose-responsive performance of GlcInsulin conjugated mRBCs (insulin-mRBCs) in vivo. To assess the blood-circulation behavior of the cell carrier, mRBCs coupled with FITC were loaded with Glc-Insulin and then i.v. injected into healthy mice. About $3 \mu \mathrm{L}$ of blood was extracted from the tail at different time points and then dispersed in $0.5 \mathrm{~mL}$ PBS with anticoagulants. Flow cytometry measurement was applied to determine the percentage of insulin-mRBC (positive in FITC) in the blood samples. The percentage of $\mathrm{mRBC}$ (injected) among total collected mRBCs appeared to be stable even $72 \mathrm{~h}$ after injection, suggesting the excellent stability of insulin-mRBCs (Figure S6A,B, Supporting Information). Liver is one of major target tissues of insulin. ${ }^{[45]}$ The liver tissues of mice treated with insulin-mRBCs or free insulin were further collected for comparison of immunofluorescence imaging. The fluorescence signals of insulin can be detected up to $4 \mathrm{~d}$ in liver after insulinmRBCs treatment; while for the mice treated with free insulin, little signal can be found at $24 \mathrm{~h}$ after injection (Figure S6C, Supporting Information). This result further substantiated the longterm insulin delivery efficiency by this RBCs system.

We then assessed the efficacy of insulin-mRBCs for treatment of hyperglycemia in STZ-induced type 1 diabetic mice. Groups of animals were treated by i.v. injection with insulin-mRBCs, free insulin plus mRBCs, Glc-Insulin alone, and PBS control. The BG levels of treated mice were then monitored over time. As shown in Figure 3A, for mice treated with free insulin plus mRBCs and with Glc-Insulin, the BG levels declined steadily to hypoglycemic within $1 \mathrm{~h}$ and recovered to hyperglycemic range $\left(\approx 500 \mathrm{mg} \mathrm{dL}^{-1}\right)$ quickly within $24 \mathrm{~h}$. In contrast, the BG level in mice treated with insulin-mRBCs decreased and maintained at about $200 \mathrm{mg} \mathrm{dL}^{-1}$ at least $24 \mathrm{~h}$ and increased gradually over $4 \mathrm{~d}$. We attributed this remarkably longer lasting impact to the stability of the mRBCs carriers in the circulation with sustained glucose-dependent insulin release.

To further understand the dynamics of insulin release in vivo, we performed intraperitoneal glucose tolerance tests (IPGTT) $1.5 \mathrm{~h}$ after administration of the insulin-mRBCs (Figure 3B). As shown, following the IPGTT, the control healthy mice exhibited a quick increase in BG level and recovery to a normal BG level within $2 \mathrm{~h}$. In diabetic mice, BG levels of insulin-mRBCs treated mice show a delayed increase in $\mathrm{BG}$ level, then decline to $\approx 200 \mathrm{mg} \mathrm{dL}^{-1}$ and maintained a normoglycemic state within $2 \mathrm{~h}$. In STZ-diabetic mice treated with free insulin + mRBCs or with Glc-Insulin, BG levels only exhibited a fleeting response. To quantitate the glucose response to the various insulin formulations, ${ }^{[15,17]}$ the area under the curve was calculated for each group. Insulin-mRBCs treated mice show enhanced responsiveness to IPGTT as compared to free insulin treated mice (Figure 3C). Furthermore, plasma insulin levels of diabetic mice were monitored over time by ELISA after IPGTT (Figure 3D). Very importantly, as BG levels increased, the serum insulin level followed closely both in the rise of glucose and on its fall to normal. This in vivo demonstration of glucose-responsive release of insulin is extremely challenging and remains elusive for most glucoseresponsive systems. Plasma glucagon levels of healthy mice, STZ-mice, and STZ-mice treated with RBC-Insulin $2 \mathrm{~h}$ were also investigated. STZ-mice had a threefold increase plasma glucagon levels compared with the nondiabetic controls, whereas RBC-Insulin treated mice had plasma glucagon levels that were similar to the nondiabetic controls (Figure S7, Supporting Information). Moreover, we compared young (from 4 weeks old mice) and old (from 1 year old mice) red blood cell vehicles for insulin delivery as well (Figure S8, Supporting Information). Interestingly, we found that $\mathrm{mRBCs}$ from young mice had a more effective and longer antidiabetes efficacy than mRBCs from old mice. This result may be attributed to the more stable and longer life span of $\mathrm{mRBCs}$ from young mice than mRBCs from aged mice in circulation. ${ }^{46,47]}$

To assess the potential for insulin-mRBCs to induce hypoglycemia, we studied their effect in healthy mice under normoglycemic conditions (Figure $3 \mathrm{E}$ ). The native insulin $+\mathrm{mRBCs}$ and Glc-Insulin treated mice showed significantly decreased BG levels compared to insulin-mRBCs treated mice. The corresponding hypoglycemia index was calculated to assess the risk of hypoglycemia. ${ }^{[15,17]}$ Treated with insulin-mRBCs significantly reduced hypoglycemic index compared to the control groups (Figure 3F).

Compared with the mouse erythrocyte, the human erythrocyte is the cell type expressing the highest level of the GLUT1, owning 500 000-700 000 molecules per cell. ${ }^{[38,48]}$ Therefore, hRBCs may have even better insulin delivery capability than mRBCs. To test this hypothesis, we examined insulin loading capacity of hRBCs. Nearly $60 \%$ of Glc-Insulin was attached to the hRBCs membrane while only $20 \%$ of Glc-Insulin was detected on mRBCs (Figure S9, Supporting Information). Furthermore, glucose-responsive insulin release from hRBCs was validated as well (Figure S9, Supporting Information). Of note, the human erythrocytes only have 2000 insulin binding sites per erythrocyte, ${ }^{[49]}$ resulting in the negligible binding amount of free insulin toward hRBCs. 
A

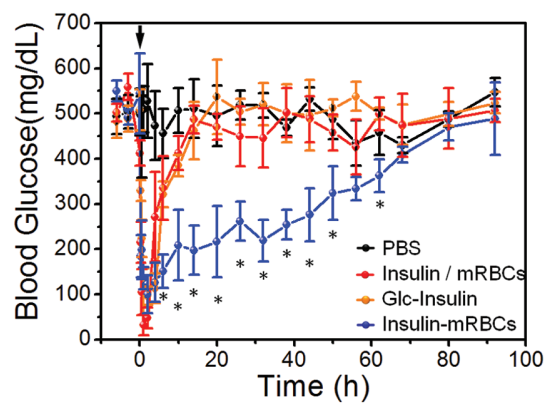

D

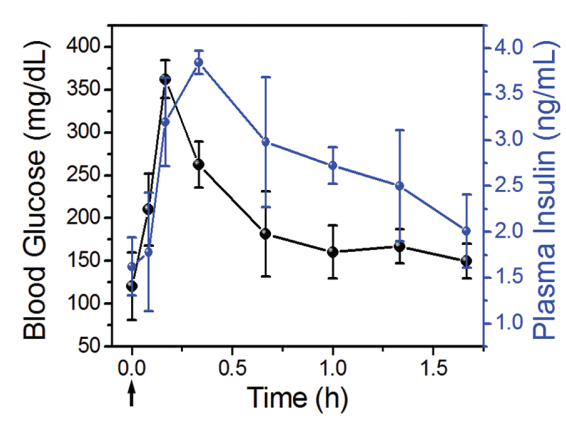

B

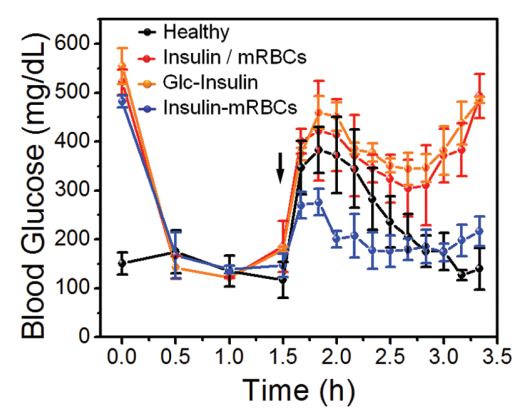

E

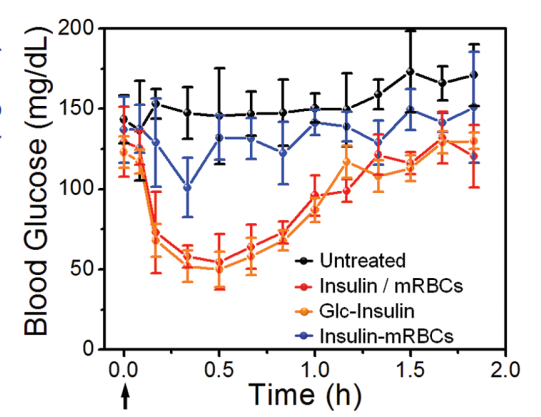

C

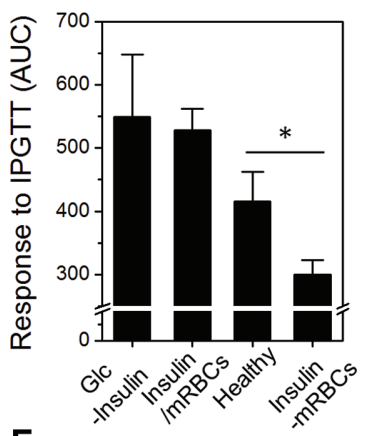

$F$

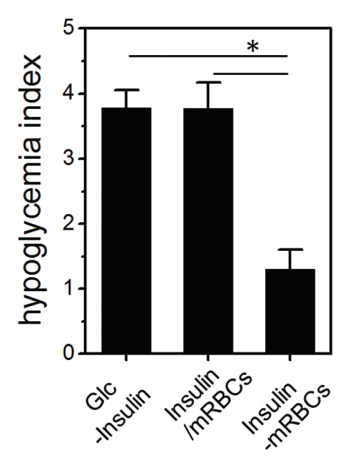

Figure 3. In vivo insulin delivery by insulin-mRBCs. A) BG levels in STZ-induced diabetic mice after treatment with free insulin + mRBCs, Glc-Insulin, insulin-mRBCs, and PBS control. The arrow indicates the administration time. B) In vivo glucose tolerance test in STZ-diabetic mice or nondiabetic mice. With the insulin-mRBCs i.v. injected at time 0 and an IPGTT performed $1.5 \mathrm{~h}$ following insulin administration. The arrow indicates the administration point. C) The responsiveness was calculated based on the area under the curve from 90 to 200 min, with the baseline set at the 90 min BC reading. D) Change in plasma insulin levels and glucose levels after IPGTT. The arrow indicates the administration point. E) BG changes of healthy mice administered by insulin-mRBCs over time. The arrow indicates the administration point. F) Quantification of the hypoglycemia index, calculated from the difference between the initial and nadir BG readings divided by the time at which nadir was reached. The error bars are based on the SD of five mice per group ( $P$-values: $* P<0.05)$.

In addition to naïve RBCs, we also synthesized a biomimetic nanoformulation using the RBC membrane-coated nanoparticles for "smart" insulin delivery in vitro and in vivo. PLGA nanoparticles coated with RBC membranes (RM) were fabricated according to the reported method (Figure 4A). ${ }^{[50]}$ The obtained RM@PLGA nanoparticles were monodispersed as revealed by the transmission electron microscopy (TEM) and dynamic light scattering (DLS). The TEM images show that most of PLGA NPs are well coated with RBC membranes with a diameter of $100 \mathrm{~nm}$ (Figure 4B). After RM coating, PLGA NPs display a slight increase in the DLS measured diameter from 145 to $160 \mathrm{~nm}$ (Figure 4C and Figure S10, Supporting Information). Western blot analysis was conducted to verify that GLUTs are present on the RM coated PLGA NPs (Figure 4D). Next, Glc-Insulin was attached to RM@PLGA NPs via the specific glucosamine-GLUT interaction as well. The attachment was also confirmed by confocal imaging and an ELISA assay (Figure S11, Supporting Information). As expected, PLGA@RM nanocarriers also release insulin in a glucose-responsive manner (Figure 4E). Further in vivo studies demonstrated that, compared with free insulin + RM@PLGA NPs, insulin-RM@PLGA NPs greatly prolongs the insulin effect to maintain BG levels and reduces the risk of hypoglycemia after injection (Figure 4F-H and Figure S12, Supporting Information).
Development of glucose-responsive formulations for insulin delivery has received considerable attentions. ${ }^{[51]}$ However, it is extremely challenging to realize a combination of fast response, long-term persistence, and excellent biocompatibility. This report demonstrates an innovative strategy using "glucoseresponsive cell" integrated with modified insulin. Unlike most existing smart insulin delivery systems that are applying synthetic materials, the formulation demonstrated in this study is simply generated by integration of RBCs and glucose derivativemodified insulin. In addition, the binding competition between glucose and glucose derivative-modified insulin bound to facilitative GLUTs offers a new and simple strategy for glucoseresponsive insulin release with fast responsiveness. We demonstrated the reversible binding between glucose derivativemodified insulin and glucose transporters activates rapidly, which enables the release of insulin from the cellular carriers to be extremely fast. The glucose-response release of insulin from the RBCs was also clearly validated through the glucose tolerance test in vivo. In the further step, this approach could be simply integrated with a painless microneedle-array patch for improving the quality of life of diabetic patients. Lastly, this strategy can be further extended to patient-centered cell therapy with therapeutics for treating a variety of diseases, with merits of both long-term sustained release and physiological signalmediated controlled release. 
A

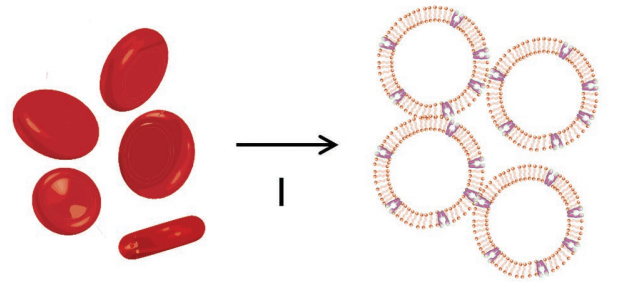

B

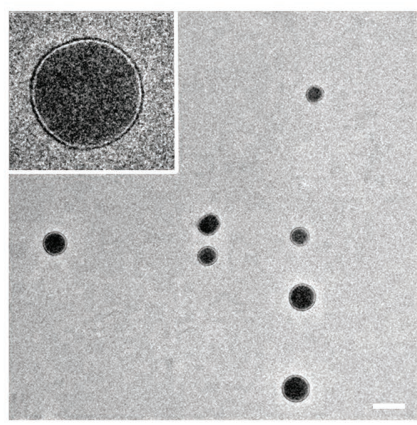

F

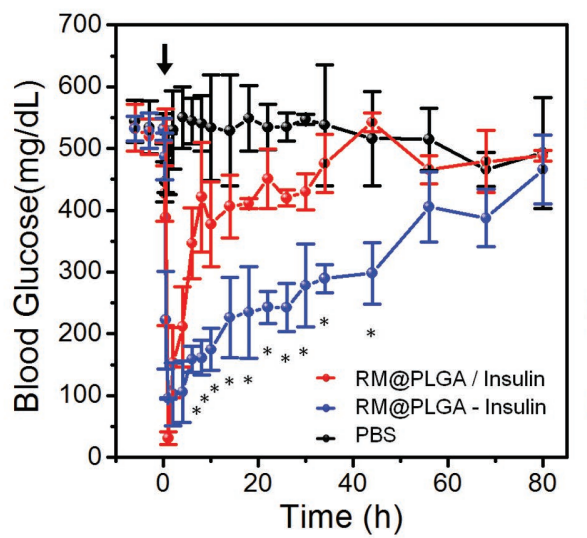

C

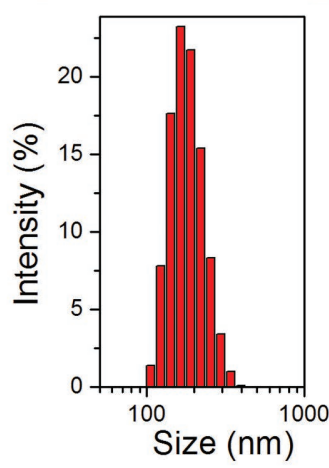

G

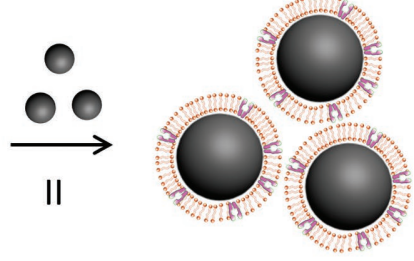

D
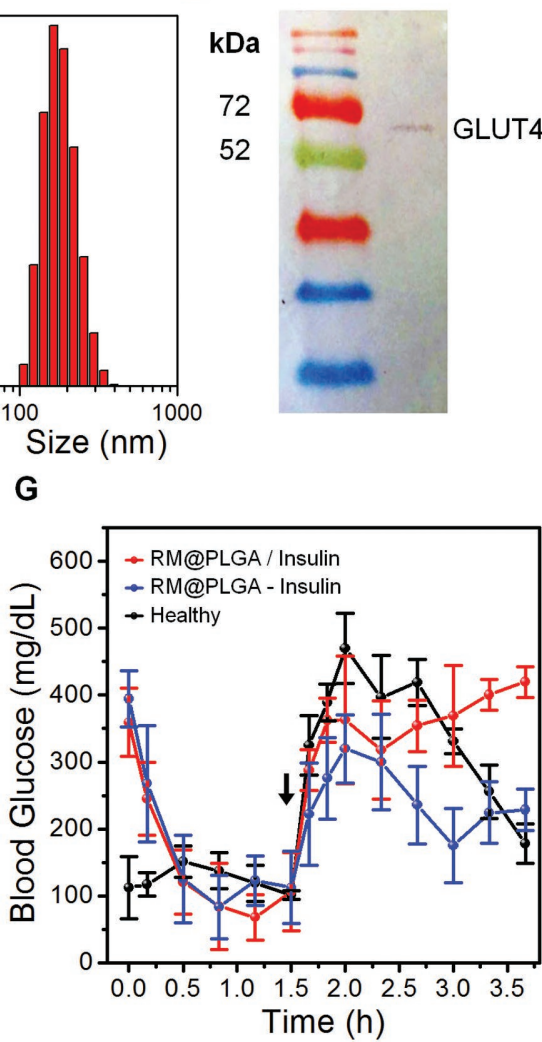

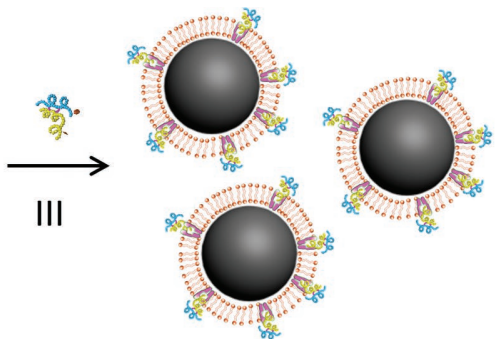

E

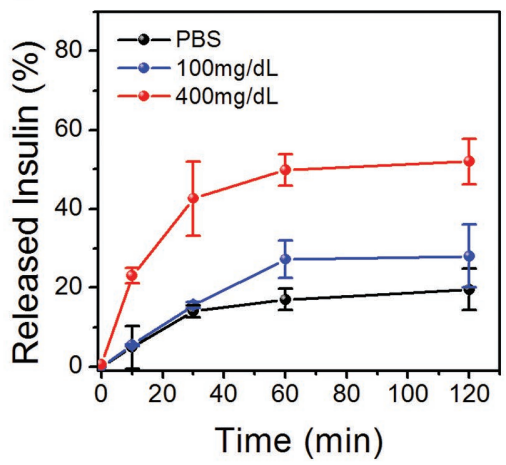

H

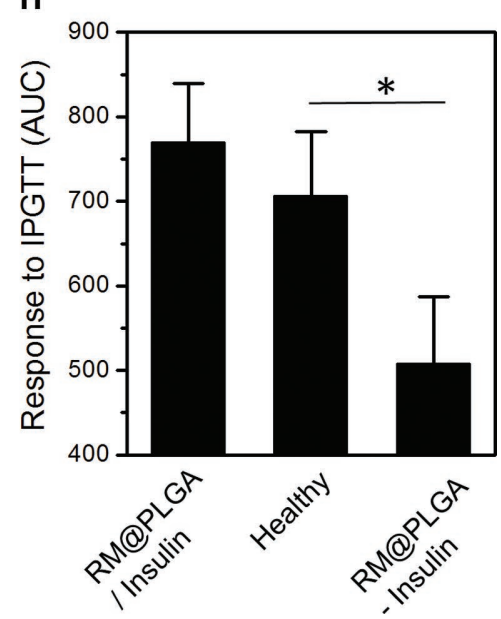

Figure 4. Glucose-responsive insulin delivery by injectable RBC-mimicking nanoparticles. A) Schematic of fabrication of insulin-PLGA@RM nanoparticles. I) Isolation of RBC membranes. II) Coating RM to PLGA nanoparticles. III) Binding of Glu-Insulun to PLGA@RM nanoparticles. B) TEM images of PLGA@RM nanoparticles. Inset: Zoom-in image (scale bar= $200 \mathrm{~nm}$ ). C) Dynamic light scattering (DLS) characterization of PLGA@RM nanoparticles. D) Western blot was conducted to verify that GLUT4 existed on the RM coated PLGA NPs. E) In vitro accumulated insulin release from the PLGA@RM in several glucose concentrations as indicated at $37^{\circ} \mathrm{C}$. F) In vivo BC levels in STZ-induced diabetic mice after treatment with free insulin + PLGA@RM NPs, insulin-PLGA@RM NPs, and PBS control. The arrow indicates the administration point. G) In vivo glucose tolerance test toward diabetic mice. With the insulin-PLGA@RM nanoparticles i.v. injected at time 0 and an IPGTT performed $1.5 \mathrm{~h}$ following insulin administration. The arrow indicates the administration point. $\mathrm{H}$ ) Responsiveness was calculated based on the area under the curve (AUC) in $120 \mathrm{~min}$, with the baseline set at the $90 \mathrm{~min}$ BG reading. The error bars are based on the standard deviation (SD) of five mice per group $(P$-values: $* P<0.05)$.

\section{Supporting Information}

Supporting Information is available from the Wiley Online Library or from the author.

\section{Acknowledgements}

This work was supported by the grants from the American Diabetes Association (ADA; 1-15-ACE-21), the Juvenile Diabetes Foundation (JDRF; 2-SRA-2016-269-A-N) to Z.G., and the grant from NC TraCS,
National Institutes of Health (NIH)'s Clinical and Translational Science Awards (CTSA, NIH grant 1UL1TR001111) at The University of North Carolina at Chapel Hill. The authors acknowledge the use of the Analytical Instrumentation Facility (AIF) at NC State, which was supported by the State of North Carolina and the National Science Foundation (NSF). The authors declare no competing financial interests.

Received: December 6, 2016 Revised: January 23, 2017 Published online: March 7, 2017 
[1] A. American Diabetes, Clin. Diabetes 2016, 34, 3

[2] L. Guariguata, D. R. Whiting, I. Hambleton, J. Beagley, U. Linnenkamp, J. E. Shaw, Diabetes Res. Clin. Pract. 2014, 103, 137.

[3] P. Raskin, E. Allen, P. Hollander, A. Lewin, R. A. Gabbay, P. Hu, B. Bode, A. Garber, Diabetes Care 2005, 28, 260.

[4] J. Pickup, H. Keen, Diabetes Care 2002, 25, 593.

[5] O. Veiseh, B. C. Tang, K. A. Whitehead, D. G. Anderson, R. Langer, Nat. Rev. Drug Discovery 2015, 14, 45.

[6] Q. Wu, L. Wang, H. Yu, J. Wang, Z. Chen, Chem. Rev. 2011, 111, 7855.

[7] A. Kowalski, Diabetes Care 2015, 38, 1036.

[8] R. Mo, T. Jiang, J. Di, W. Tai, Z. Gu, Chem. Soc. Rev. 2014, 43, 3595.

[9] A. J. Vegas, O. Veiseh, M. Gürtler, J. R. Millman, F. W. Pagliuca, A. R. Bader, J. C. Doloff, J. Li, M. Chen, K. Olejnik, Nat. Med. 2016, 22, 306.

[10] C. Martin, Nat. Nanotechnol. 2015, 10, 910.

[11] K. Podual, F. J. Doyle, N. A. Peppas, J. Controlled Release 2000, 67, 9.

[12] K. Podual, F. J. Doyle, N. A. Peppas, Polymer 2000, 41, 3975.

[13] Z. Gu, T. T. Dang, M. Ma, B. C. Tang, H. Cheng, S. Jiang, Y. Dong, Y. Zhang, D. G. Anderson, ACS Nano 2013, 7, 6758.

[14] Z. Gu, A. A. Aimetti, Q. Wang, T. T. Dang, Y. Zhang, O. Veiseh, H. Cheng, R. S. Langer, D. G. Anderson, ACS Nano 2013, 7, 4194.

[15] J. Yu, Y. Zhang, Y. Ye, R. DiSanto, W. Sun, D. Ranson, F. S. Ligler, J. B. Buse, Z. Gu, Proc. Natl. Acad. Sci. USA 2015, 112, 8260.

[16] M. Brownlee, A. Cerami, Science 1979, 206, 1190.

[17] D. H.-C. Chou, M. J. Webber, B. C. Tang, A. B. Lin, L. S. Thapa, D. Deng, J. V. Truong, A. B. Cortinas, R. Langer, D. G. Anderson, Proc. Natl. Acad. Sci. USA 2015, 112, 2401.

[18] F.-L. Mi, Y.-Y. Wu, Y.-H. Lin, K. Sonaje, Y.-C. Ho, C.-T. Chen, J.-H. Juang, H.-W. Sung, Bioconjugate Chem. 2008, 19, 1248.

[19] K. Kataoka, H. Miyazaki, M. Bunya, T. Okano, Y. Sakurai, J. Am. Chem. Soc. 1998, 120, 12694.

[20] W. Wu, N. Mitra, E. C. Yan, S. Zhou, ACS Nano 2010, 4, 4831.

[21] H. Kim, Y. J. Kang, S. Kang, K. T. Kim, J. Am. Chem. Soc. 2012, 134, 4030.

[22] X. Hu, J. Yu, C. Qian, Y. Lu, A. R. Kahkoska, Z. Xie, X. Jing, J. B. Buse, Z. Gu, ACS Nano 2017, 11, 613.

[23] C. Wang, X. Sun, L. Cheng, S. Yin, G. Yang, Y. Li, Z. Liu, Adv. Mater. 2014, 26, 4794.

[24] A. C. Anselmo, V. Gupta, B. J. Zern, D. Pan, M. Zakrewsky, V. Muzykantov, S. Mitragotri, ACS Nano 2013, 7, 11129.

[25] V. R. Muzykantov, Expert Opin. Drug Delivery 2010, 7, 403.

[26] C. H. Villa, D. C. Pan, S. Zaitsev, D. B. Cines, D. L. Siegel, V. R. Muzykantov, Ther. Delivery 2015, 6, 795.

[27] R. Mukthavaram, G. Shi, S. Kesari, D. Simberg, J. Controlled Release 2014, 183, 146.
[28] Z. Wu, T. Li, J. Li, W. Gao, T. Xu, C. Christianson, W. Gao, M. Galarnyk, Q. He, L. Zhang, ACS Nano 2014, 8, 12041.

[29] X. Sun, C. Wang, M. Gao, A. Hu, Z. Liu, Adv. Funct. Mater. 2015, 25, 2386.

[30] J. Li, F. K. Ma, Q. F. Dang, X. G. Liang, X. G. Chen, Front. Mater. Sci. 2014, 8, 363.

[31] X. Y. Jiang, H. L. Xin, J. J. Gu, F. Y. Du, C. L. Feng, Y. K. Xie, X. L. Fang, J. Pharm. Sci. 2014, 103, 1487.

[32] A. Luciani, J. C. Olivier, O. Clement, N. Siauve, P. Y. Brillet, B. Bessoud, F. Gazeau, I. F. Uchegbu, E. Kahn, G. Frija, C. A. Cuenod, Radiology 2004, 231, 135.

[33] M. Zhang, Z. Zhang, D. Blessington, H. Li, T. M. Busch, V. Madrak, J. Miles, B. Chance, J. D. Glickson, G. Zheng, Bioconjugate Chem. 2003, 14, 709.

[34] C. Dufes, A. G. Schatzlein, L. Tetley, A. I. Gray, D. G. Watson, J. C. Olivier, W. Couet, I. F. Uchegbu, Pharm. Res. 2000, 17, 1250.

[35] L. Speizer, R. Haugland, H. Kutchai, Biochim. Biophys. Acta 1985, 815, 75.

[36] Q. L. He, I. Minn, Q. Wang, P. Xu, S. A. Head, E. Datan, B. Yu, M. G. Pomper, J. O. Liu, Angew. Chem., Int. Ed. Engl. 2016, 55, 12035.

[37] M. Mueckler, Eur. J. Biochem. 1994, $219,713$.

[38] I. Vrhovac, D. Breljak, I. Sabolić, Period. Biol. 2014, 116, 131.

[39] A. Montel-Hagen, M. Sitbon, N. Taylor, Curr. Opin. Hematol. 2009, 16, 165.

[40] A. Montel-Hagen, L. Blanc, M. Boyer-Clavel, C. Jacquet, M. Vidal, M. Sitbon, N. Taylor, Blood 2008, 112, 4729.

[41] M. Uldry, M. Ibberson, M. Hosokawa, B. Thorens, FEBS Lett. 2002, 524, 199.

[42] D. Ranganathan, S. Thamake, I. Tworowska, E. Delpassand, J. Nucl. Med. 2014, 55, 1004.

[43] A. Chopra, Molecular Imaging and Contrast Agent Database (MICAD), National Center for Biotechnology Information (US), Bethesda, MD 2004.

[44] A. V. Korotcov, Y. Ye, Y. Chen, F. Zhang, S. Huang, S. Lin, R. Sridhar, S. Achilefu, P. C. Wang, Mol. Imaging Biol. 2012, 14, 443.

[45] M. Lu, M. Wan, K. F. Leavens, Q. Chu, B. R. Monks, S. Fernandez, R. S. Ahima, K. Ueki, C. R. Kahn, M. J. Birnbaum, Nat. Med. 2012, $18,388$.

[46] D. Shemin, D. Rittenberg, J. Biol. Chem. 1946, 166, 627.

[47] W. H. Crosby, Blood 1959, 14, 399.

[48] D. Anstee, Vox Sang. 2011, 100, 140

[49] K. K. Gambhir, J. A. Archer, C. J. Bradley, Diabetes 1978, 27, 701.

[50] C.-M. J. Hu, L. Zhang, S. Aryal, C. Cheung, R. H. Fang, L. Zhang, Proc. Natl. Acad. Sci. USA 2011, 108, 10980.

[51] Y. Lu, A. A. Aimetti, R. Langer, Z. Gu, Nat. Rev. Mater. 2016, 1, 16075. 